\title{
Esporte, stress e burnout
}

\author{
Sport, stress, and burnout
}

\author{
Carlos Eduardo Lopes VERARDI' \\ Maria Cristina de Oliveira Santos MIYAZAKI ${ }^{1}$ \\ Kazuo Kawano NAGAMINE' \\ Ana Paula da Silva LOBO' \\ Neide Aparecida Micelli DOMINGOS
}

\begin{abstract}
Resumo
Para muitos autores, o stress está negativamente relacionado ao desempenho individual. Algumas evidências, entretanto, sugerem que a performance em determinada tarefa será mais baixa sob níveis muito reduzidos ou muito elevados de stress, e ótima sob níveis moderados de stress. O burnout está associado à percepção de que os recursos pessoais são insuficientes ou inadequados para enfrentar o stress. Identificar estressores e estratégias de enfrentamento que, se empregadas pelos indivíduos, conseguem amortecer o impacto do stress, permite delinear programas preventivos e de intervenção. O objetivo deste estudo é apresentar uma revisão atualizada sobre burnout contexto esportivo.
\end{abstract}

Unitermos: Burnout. Esporte. Exercício. Stress. Stress profissional.

\begin{abstract}
For many authors, stress is negatively related to individual performance. Some evidence, however, suggests that performance in a given task will be lower undervery low orvery high levels of stress and great under moderate levels of stress. Burnout is associated with the perception that personal resources are insufficient or inadequate to cope with stress. Identifying stressors and coping strategies which, if used by individuals, can minimize the impact of stress, and allow preventive and intervention programs to be designed. The aim of this study is to present an updated review of burnout within sporting context.
\end{abstract}

Uniterms: Burnout. Sport. Exercise. Stress. Occupational stress.

Atualmente há grande preocupação em proporcionar condições adequadas de trabalho, visando não só à produtividade do trabalhador, mas também à sua qualidade de vida, à sua saúde e ao seu futuro. Nesse sentido, o esporte profissional está associado a uma permanente busca da excelência física, técnica, tática e psicológica e, ao mesmo tempo, a uma preocupação em evitar ou manejar problemas que retardem ou interrompam a vida profissional do atleta.

O stress associado à competição esportiva é um tópico altamente relevante, relacionado principalmente ao desempenho de atletas profissionais, submetidos aos rigores de treinamento e às demandas de contextos competitivos (Hanton, Thomas \& Mellalieu, 2008). Stress

\footnotetext{
$\boldsymbol{\nabla} \mathbf{\nabla} \boldsymbol{\nabla}$

1 Faculdade de Medicina de São José do Rio Preto, Faculdade de Medicina. Av. Brigadeiro Faria Lima, 5416, VI. São Pedro, 15090-000, São José do Rio Preto, SP, Brasil. Correspondência para/Correspondência to: C.E.L. VERARDI. E-mail: <celverardi@hotmail.com>.
} 
e estratégias de enfrentamento têm sido alvo crescente de estudos relacionados à prática esportiva, uma vez que enfrentar adequadamente o stress é parte das habilidades requeridas de um atleta (Nicholls, Jones, Polman \& Borkoles, 2009; Samulski \& Lopes, 2008; Thatcher \& Day, 2008).

O objetivo deste estudo é apresentar uma revisão sobre stress, burnout e enfrentamento no contexto esportivo. Foram pesquisadas as bases de dados Scopus, PubMed, Psyclnfo, Lilacs e MedLine, no período entre 2004 e 2009, utilizando como palavras-chave estresse, burnout, adaptação psicológica, esporte e seus similares na língua inglesa. Foram ainda utilizados livros, dissertações e teses sobre o tema (Coordenação de Aperfeiçoamento de Pessoal de Nível Superior, 2009).

\section{Estresse estratégias de enfrentamento em atletas}

O termo stress é utilizado por diferentes áreas do conhecimento, com significados diversos. Alguns aspectos essenciais, entretanto, estão envolvidos quando se discute stress: 1) uma causa interna ou externa, denominada estressor; 2) uma avaliação sobre aspectos potencialmente ameaçadores (ou não) da situação; 3) respostas de enfrentamento e 4) efeitos psicofisiológicos ou reações ao stress (Lazarus, 1993).

Stress é uma "experiência emocional negativa, acompanhada por mudanças bioquímicas, fisiológicas, cognitivas e comportamentais previsíveis, que visam alterar o evento estressor ou acomodar seus efeitos" Taylor (2009, p.147). Lipp (2003, p.18) define stress"como uma reação psicofisiológica muito complexa que tem em sua gênese a necessidade do organismo fazer face a algo que ameace sua homeostase interna".

O termo pode ainda ser compreendido como um estímulo ou evento ambiental causador de tensão, denominado estressor; como resposta ou reação individual ao estressor, incluindo componentes psicológicos e fisiológicos; e como processo ou relação entre indivíduo e ambiente. Quando visto como processo, o indivíduo"é um agente ativo que pode influenciar o impacto do estressor por meio de estratégias comportamentais, cognitivas e emocionais" (Sarafino, 2008, p.62).

É importante ressaltar que é a percepção que o indivíduo tem acerca de um evento, e não o evento em si, que determina seu potencial como estressor. Assim, uma mesma experiência pode ser percebida como estressora por uma pessoa e como inócua por outra (Lazarus, 1993; Marques \& Rosado, 2005; Sarafino, 2008; Taylor, 2009). Quando algo é percebido como estressor, entretanto, tende a desencadear respostas fisiológicas semelhantes nos indivíduos (Kavanagh, 2005). Apesar de a ênfase ser dada às emoções negativas associadas ao stress, emoções positivas podem ocorrer concomitan-temente (Folkman \& Moskowitz, 2004).

Embora o stress com frequência esteja negativamente associado ao desempenho individual, evidências sugerem que a "performance em determinada tarefa será mais baixa em níveis muito reduzidos ou muito elevados de estresse e ótima em níveis moderados de estresse" (Kavanagh, 2005, p.16). Assim, o stress moderado pode melhorar o desempenho porque há estímulo para manter a vigilância do indivíduo, mas não um excesso de estímulo para distraí-lo de seu objetivo. Quando o nível de stress é baixo, o indivíduo pode não estar suficientemente alerta para ter um desempenho adequado; quando é muito alto, por outro lado, a excitação excessiva pode prejudicar o desempenho. Vários modelos vêm sendo propostos para explicar o desempenho humano em condições de stress (Kavanagh, 2005).

É inegável, entretanto, que o stress faz parte da vida das pessoas e que, quando excessivo, pode ter consequências negativas. Identificar estressores e estratégias de enfrentamento que, se empregadas pelos indivíduos, conseguem amortecer o seu impacto, permite delinear programas preventivos e de intervenção. Estudos com esse objetivo têm sido realizados em diferentes áreas do conhecimento, com diferentes objetivos e participantes, inclusive atletas.

Enfrentamento (coping) é o termo utilizado para designar o processo de lidar com demandas (internas ou externas), avaliadas pelo indivíduo como superiores a seus recursos ou possibilidades (Lazarus \& Folkman, 1984; Taylor \& Stanton, 2007).

Lazarus e Folkman (1984, p.141) propuseram a definição provavelmente mais conhecida de enfrentamento: "esforços para mudanças comportamentais e cognitivas constantes para lidar com demandas específicas internas e/ou externas, avaliadas pelo indivíduo como excessivas ou além de suas possibilidades". Esses esforços influenciam e são influenciados pelo ambiente. 
As estratégias de enfrentamento, utilizadas pelos indivíduos para lidar com o stress, têm sido classificadas como focalizadas na emoção ou no problema (Nicholls \& Polman, 2007; Straub, 2005). Estratégias focalizadas na emoção são habitualmente utilizadas quando as pessoas acreditam que pouco ou nada pode ser feito para alterar uma situação. Incluem fuga-evitação, distanciamento e reavaliação positiva da situação. Podem ser positivas ou negativas, como busca de suporte social, distração e uso de álcool (Straub, 2005). Quando o enfrentamento é focado no problema, "as pessoas lidam diretamente com a situação estressante, seja reduzindo as demandas ou aumentando a capacidade de lidar com o estressor" (Straub, 2005, p.153).

Enfrentamento é, portanto, uma resposta individual de adaptação a situações consideradas difíceis. Mudanças no estilo de vida ou problemas associados a emoções negativas (exemplo a ansiedade e a depressão) requerem novo repertório de comportamentos, diferente daquele utilizado anteriormente. Esse novo repertório inclui as estratégias de enfrentamento utilizadas para lidar com as novas demandas. Já foram identificados alguns recursos de enfrentamento que auxiliam a manejar o stress e a reduzir o sofrimento, e que estão associados a melhores resultados em termos de saúde. Tais recursos incluem o otimismo, a percepção de controle, a autoestima e o suporte social (Taylor \& Stanton, 2007).

Analisando várias definições de enfrentamento, Gimenez (1997, p.116) concluiu que a maioria delas compartilha a noção de que "as pessoas ativamente às exigências impostas a elas" e associam enfrentamento a stress. Assim, enfrentamento será aqui compreendido não como uma característica pessoal, mas como "esforços para lidar com situações estressantes que mudam ao longo do tempo... delineados a partir de contextos particulares que exigem adaptação" (Gimenez, 1997, p.17).

O enfrentamento de determinada situação depende de uma avaliação individual acerca da mesma. Essa avaliação pode identificar perdas, danos, ameaça de perdas ou desafio. As respostas de enfrentamento podem ser uma ação direta ou a solução do problema, reduzindo o stress e a emoção negativa a ele associada. Quando um problema não pode ser solucionado por meio de uma ação direta, estratégias cognitivas podem ser utilizadas, sempre considerando que"... ações e pensamentos [não] são mutuamente exclusivos, ao contrário, processos cognitivos estão presentes em ações diretas particulares e estas, sem dúvida, também presentes durante as avaliações das pessoas" (Gimenez, 1997, p.126).

As estratégias de enfrentamento utilizadas por atletas, portanto, variam em função da percepção individual do stress (Anshel \& Si, 2008). No contexto esportivo, a percepção individual do stress varia em função do que está "em jogo" (exemplo o título), de crenças individuais acerca do próprio posicionamento no ranking de determinado campeonato e dos recursos individuais (exemplo o preparo e a forma física) (Thatcher \& Day, 2008). "... atletas de sucesso serão, certamente, aqueles que melhores capacidades demonstram na gestão e no controle das situações potencialmente estressantes" (Marques \& Rosado, 2005, p.71).

Ivarsson (2008) identificou ansiedade, falta de confiança, stresse estratégias pouco efetivas de enfrentamento como importantes fatores que aumentam a vulnerabilidade para lesões em jogadores de futebol de elite. Outras fontes de stress entre jogadores de futebol incluem ansiedade em relação ao próprio desempenho, ausência de feedback, dificuldade para conciliar o esporte com outras atividades (exemplo o estudo), deficit de suporte social formal (exemplo o manager, o programa de trabalho e o psicólogo do esporte) e/ou informal, como capacidade de concentração e insegurança em relação ao trabalho como atleta profissional (Noblet \& Gifford, 2002).

Estudo com jogadores de basquetebol de alta competição indicou que as fontes de stress, para esses atletas, provinham dos diversos aspectos envolvidos na prática competitiva, como viagens, questões financeiras, pessoais, participação em esporte coletivo e de relacionamento interpessoal (Marques \& Rosado, 2005).

O impacto do stress sobre atletas inclui prejuízos como rigidez no desempenho e no raciocínio, redução na habilidade de analisar situações complexas e de manipular informações de forma adequada, dificuldade para concluir tarefas e redução da acurácia. Além dos efeitos individuais, o stress pode afetar de forma negativa o funcionamento do indivíduo em atividades de grupo, com redução da qualidade de comunicação e com tomada de decisões incorretas (Kavanagh, 2005). A 
exposição prolongada a determinados estressores pode ter consequências negativas graves, como a exaustão profissional (Taylor, 2009).

De acordo com Weinberg e Gould (2008), "treinamento excessivo" (overtrainning) refere-se a um ciclo curto de treinamento, durante o qual os atletas são submetidos a uma sobrecarga excessiva, próxima de sua capacidade máxima. A exaustão é o resultado final do treinamento excessivo, uma condição de fadiga e baixo rendimento, com dificuldade para manter o regime de treinamento e os resultados de desempenho padrão, associada a baixa recuperação. Burnout, por sua vez, é uma resposta psicofisiológica, mais completa, de afastamento do treinamento excessivo e das demandas competitivas. A queda no rendimento pode ser um sinal do acometimento do burnout, e, entre outras causas que podem levar o atleta à desistência do esporte (dropout), estão a estafa e o treinamento excessivo. Hipoteticamente, a resposta ao treinamento excessivo e à estafa, às vezes, resultará em burnout, sendo este decorrente daqueles fenômenos (Weinberg \& Gould, 2008).

Para atletas profissionais, em longo prazo, o desequilíbrio entre as exigências da profissão e as estratégias de enfrentamento utilizadas pode levar ao abandono da carreira (Marques \& Rosado, 2005).

Entretanto, é importante ressaltar que estratégias adequadas de enfrentamento podem ser ensinadas, quando necessário. Quando estratégias adequadas não estão disponíveis ou não são desenvolvidas, altos níveis de stress podem acarretar exaustão, baixo comprometimento com o grupo ou com a organização profissional, rotatividade e até transtornos psiquiátricos, como a síndrome do esgotamento profissional ou burnout (Brasil, 2001; Kavanagh, 2005).

Para jogadores profissionais e para outras atletas, a síndrome do esgotamento profissional ou burnout está associada a lesões mais frequentes. Além disso, jogadores com maior experiência e maior número de vitórias relatam mais stress e exaustão (Cresswell \& Eklund, 2005a).

\section{Burnout, enfrentamento e esporte}

O stress ocupacional pode ter um impacto nega-

308 tivo sobre a saúde, o bem-estar e o rendimento pro- fissional das pessoas (Brasil, 2001; Maslach \& Leiter, 2008; Sarafino, 2008; Taylor, 2009).

Síndrome do esgotamento profissional, sensação de estar acabado ou síndrome de burnout são termos utilizados para denominar "um tipo de resposta prolongada a estressores emocionais e interpessoais crônicos no trabalho" (Brasil, 2001, p.191). O burnoutestá associado a situações profissionais socialmente complexas, que envolvem a representação que o indivíduo possui acerca de si mesmo e do outro. Quando ocorre, mesmo pessoas intensamente envolvidas com o seu trabalho desinteressam-se dele e percebem como inútil qualquer esforço para realizá-lo (Brasil, 2001).

Maslach e Jackson (1981, p.99) definiram burnout como"uma síndrome de exaustão emocional e cinismo que ocorre frequentemente entre indivíduos que trabalham com seres humanos". Quando nesse estado, as pessoas acreditam ter exaurido seus recursos psicológicos, apresentam atitudes cínicas e negativas em relação ao outro e uma avaliação negativa de si próprias. Sentem-se, portanto, "insatisfeitas consigo mesmas e com seu rendimento no trabalho" (Maslach \& Jackson, 1981, p.99). Além disso, burnout parece associado a "... sofrimento emocional, inclusive exaustão física, insônia, uso crescente de álcool e drogas e problemas conjugais e familiares" (Maslach \& Jackson, 1981, p.100).

De natureza multidimensional, o esgotamento profissional envolve três componentes básicos: 1) exaustão emocional, com sentimentos de extrema fadiga e percepção de incapacidade para"se doar" afetivamente; 2) despersonalização, com o desenvolvimento de atitudes cínicas e sentimentos negativos e 3) insatisfação com a própria realização profissional, com tendência a se avaliar negativamente, especialmente na relação com os usuários ou clientes, com sentimentos de infelicidade e insatisfação com as próprias realizações no trabalho (Maslach \& Jackson, 1981).

No contexto esportivo, burnout é discutido, principalmente, como uma resposta do indivíduo ao stress crônico, caracterizada por redução no desempenho, desvalorização do esporte e exaustão física e emocional. Publicações na mídia, a respeito de "experiências esportivas negativas de uma série de atletas famosos" (Cresswell \& Eklund, 2006a, p.219) deram início ao interesse por pesquisas nessa área. Os pesquisadores, 
por sua vez, propuseram que as experiências negativas desses atletas eram semelhantes ao burnout experimentado por profissionais de outras áreas (Cresswell \& Eklund, 2006a; 2006b).

Para vários pesquisadores em psicologia do esporte, as características do burnout em atletas são semelhantes às experimentadas por profissionais que atuam em contato direto e frequente com outros seres humanos. A exaustão profissional ou burnout está associada à percepção de que os recursos pessoais são insuficientes ou inadequados para enfrentar o stress. Como decorrência, o indivíduo desenvolve atitudes, condutas e sentimentos negativos, que acarretam problemas de ordem prática e emocional no ambiente de trabalho. Inúmeros relatos de atletas de elite que abandonaram o esporte no auge de suas carreiras são considerados, para muitos autores, exemplos dos efeitos do stress excessivo no contexto esportivo (Ivarsson, 2008; Lemyre, Hall \& Roberts, 2008; Raedeke, 1997).

Embora a definição de burnout de Maslach e Jackson (1981) seja amplamente reconhecida, foi elaborada para ocupações em que a relação entre profissional e cliente é fundamental, como, por exemplo, os serviços de saúde. Quando utilizada em outros contextos, é preciso considerar dimensões específicas, como, por exemplo, objetivos e responsabilidades inerentes a cada profissão.

No caso de atletas, embora a relação atleta/técnico seja importante, não é o elemento fundamental do contexto esportivo. No esporte, portanto, burnout precisa ser definido em função do desempenho esportivo, que é o elemento fundamental da prática desses profissionais (Raedeke, 1997). Para atletas, a exaustão profissional pode ser decorrente das demandas do treinamento e das competições, e pode estar associada à "... percepção de baixo desempenho profissional em termos de habilidades e rendimento esportivo" (Raedeke, 1997, p.397).

A exaustão profissional em atletas apresenta três importantes aspectos: 1) relato de esgotamento emocional e físico, decorrente das demandas excessivas impostas por treinos e competições; 2) reduzido senso de autorrealização, com a percepção de que os objetivos são inatingíveis e 3) desvalorização e desinteresse pelo esporte (Gustafsson, Hassmén, Kentta \& Johansson, 2008; Lemyre et al., 2008; Raedeke, 1997).
Das três dimensões de burnout (exaustão emocional, despersonalização e insatisfação com o próprio desempenho), a despersonalização parece ser o aspecto menos aplicável a atletas, provavelmente pelas diferenças contextuais entre esporte e prestação de serviços a outras pessoas. Uma vez que a despersonalização representa a desvalorização e o desinteresse para o que é importante, nesse domínio particular, despersonalização para atletas pode representar o desenvolvimento de atitudes negativas no seu envolvimento com o esporte (Raedeke, 1997).

Explicações para o burnout no contexto esportivo estão frequentemente associadas ao stress, embora nem todos aqueles que apresentam stress desenvolvam burnout. A falta de controle que muitos atletas sentem em relação à própria vida, assim como a necessidade de fazer esporte para sobreviver, são algumas explicações sugeridas para explicar burnout em atletas. $\mathrm{O}$ conhecimento nessa área, entretanto, ainda é limitado.

Um estudo qualitativo sobre burnout em atletas suecos de elite, de diferentes modalidades esportivas, identificou três temas associados e suas subcategorias: 1) sintomas e consequências: falta de realização, exaustão, desvalorização da participação no esporte, manifestações motivacionais, enfrentamento e manifestações cognitivo-afetivas; 2) fatores associados ao burnout: relacionados ao esporte e treinamento, estressores psicossociais e personalidade e 3 ) fatores de restrição, isto é, aqueles que impedem o atleta de abandonar o esporte apesar dos problemas enfrentados: esporte como identidade e autoestima e restrições. Esses dados, embora obtidos no contexto esportivo da Suécia, apontam a natureza multifatorial do burnout no esporte (Gustafsson et al., 2008).

Perfeccionismo é um fator frequentemente associado à exaustão profissional e que parece aumentar a vulnerabilidade para burnout. O perfeccionismo pode ser: 1) orientado para o outro, com um exagerado nível de exigência em relação ao comportamento do outro; 2) auto-orientado, com padrões autoimpostos excessivamente altos e irreais de desempenho, autocrítica exagerada e acentuada dificuldade para aceitar qualquer tipo de falta cometida e 3) socialmente orientado, com crença na necessidade de sempre atender às expectativas dos outros (que acredita serem extremamente elevadas) para ser aceito. 
Quando orientado para o outro, o perfeccionismo parece ter função adaptativa, na medida em que impulsiona o indivíduo a buscar um desempenho cada vez melhor nas tarefas que executa. Entretanto, está também associado a uma autoavaliação extremamente rígida, com alto nível de exigência e uma tendência para centrar-se nos aspectos negativos do próprio desempenho.

No perfeccionismo socialmente orientado, por sua vez, o indivíduo busca manter a aprovação dos outros por meio de um desempenho perfeito, havendo preocupação excessiva acerca de cometer erros, além de altas expectativas.

Um estudo com jogadores de futebol concluiu que dimensões negativas do perfeccionismo parecem antecedentes do burnout nesses atletas. Perfeccionismo auto-orientado e socialmente orientado parecem aumentar a vulnerabilidade para a exaustão nesses atletas (Hill, Hall, Appleton \& Kozub, 2008). A presença de suporte social, entretanto, parece ser um importante fator protetor do burnout em atletas (Cresswell, 2009).

De acordo com Pires, Brandão e Machado (2005), a exaustão profissional ainda é pouco identificada no contexto esportivo, pelo fato de os sintomas de ordem física e psicológica não serem reconhecidos em curto prazo. Em muitos casos, atletas estão vulneráveis ao burnout a partir do momento em que surge a incompatibilidade com planos e metas iniciais na modalidade esportiva, por vivenciarem frequentes cobranças e críticas, cansaço e stress, cuja consequência principal é o abandono da prática esportiva. Muitos atletas jovens desistem ou abandonam a carreira esportiva quando atingem o nível profissional. Antecedendo a desistência, eles passam por um estágio de esgotamento, causado por motivos físicos, psicológicos ou sociais.

Para Samulski (2002, p.349), ao vivenciar o burnout, o afastamento do ambiente estressor torna-se inevitável. No contexto esportivo, entretanto, burnout difere do abandono da prática esportiva"porque envolve algumas características psicológicas e exaustão emocional, respostas negativas dos colegas, diminuição da autoestima e depressão". Assim, atletas abandonam o esporte por várias razões, sendo burnout uma delas. A exaustão profissional pode acometer qualquer atleta que pratica esporte com certa intensidade competitiva. Portanto, presença, mas sim as circunstâncias esportivas e as fontes de stress associadas à prática esportiva habitual, como intensidade competitiva, independentemente do nível esportivo, percepção da presença de estressores no contexto esportivo, atitudes de treinadores e/ou familiares, quantidade e intensidade das competições (Garcés de Los Fayos \& Vives Benedicto, 2002).

Algumas variáveis associadas ao burnout no contexto esportivo incluem estilo do treinador, altas demandas competitivas, estratégias de enfrentamento, estilo de vida externo, monotonia do treinamento e escassez de reforços positivos. Considerando tais variáveis, pesquisadores têm sugerido programas de prevenção e intervenção no contexto esportivo, focando aspectos como estruturar de maneira mais racional o trabalho desportivo, planificar períodos de prática mental e treinamento psicológico, modificar a estrutura social do esporte competitivo e aumentar a idade mínima exigida para a prática esportiva e competitiva (Garcés de Los Fayos \&Vives Benedicto, 2002; Orlick, 2008).

\section{Burnout: possibilidade de avaliação}

Tradicionalmente, o instrumento mais utilizado para avaliar burnout tem sido o Inventário Maslach de Burnout (Maslach Burnout Inventory ou MBI), projetado para avaliar vários aspectos dessa síndrome em profissionais da área de prestação de serviços humanos (Maslach \& Jackson, 1981). Um instrumento psicométrico específico para a mensuração de burnout em atletas, 0 Athlete Burnout Questionnaire (ABQ), desenvolvido por Raedeke e Smith (2001), tem sido considerado mais adequado para investigar burnout no contexto esportivo.

O Athlete Burnout Questionaire é composto por 15 itens e três subescalas, que avaliam a frequência de sentimentos relativos ao burnout: exaustão física e emocional (exemplo: eu estou exausto pelas demandas físicas e emocionais do esporte); reduzido senso de realização esportiva (exemplo: não importa o que eu faço, eu não executo como devo); desvalorização da modalidade esportiva (exemplo: eu tenho sentimentos negativos em relação ao esporte). As respostas são dadas em uma escala tipo Likert: 1) quase nunca; 2) raramente; 3) algumas vezes; 4) frequentemente e 5) quase sempre. 
Cresswell e Eklund (2006b) avaliaram a validade do Maslach Burnout Inventory - General Survey (MBI-GS), versão adaptada do MBI, e do ABQ. Concluíram que, apesar de as duas medidas apresentarem algumas limitações, de forma geral ambas apresentam validade satisfatória. Sugeriram ainda que pesquisas em contextos esportivos devem utilizar o ABQ (Raedeke \& Smith, 2001), visando estabelecer normas e possíveis consequências do burnout em atletas. Pires, Brandão e Silva (2006) traduziram o ABQ como Questionário de Burnout para Atletas (QBA). Os resultados obtidos confirmaram tanto a validade do construto quanto sua confiabilidade.
Muitos estudos (Quadro 1) têm observado a frequência da experiência de sentimentos relacionados ao burnout em várias modalidades esportivas, com atletas profissionais e amadores de ambos os sexos, utilizando o instrumento desenvolvido por Raedeke e Smith (2001), o ABQ.

Além de elaborar e avaliar programas preventivos e de intervenção, é também altamente relevante identificar precocemente sinais de risco para burnout em atletas. Esses sinais incluem, por exemplo, persistência de sentimentos de decepção e mudanças de humor, como precursores da exaustão emocional (Cresswell \& Eklund, 2004).

Quadro 1. Síntese de estudos que utilizaram o Athlete Burnout Questionnaire em diferentes modalidades esportivas.

\begin{tabular}{|c|c|c|c|}
\hline Estudos & Objetivo & Descrição da amostra estudada & Descobertas \\
\hline $\begin{array}{l}\text { Cresswell e } \\
\text { Eklund (2004) }\end{array}$ & $\begin{array}{l}\text { Avaliar a validade de uma pro- } \\
\text { posta de sinais prematuros e } \\
\text { suas correlações com as prin- } \\
\text { cipais características de burnout }\end{array}$ & $\begin{array}{l}\text { Jogadores amadores e profis- } \\
\text { sionais de rugby }(n=199) \text { entre } 19 \text { e } \\
33 \text { anos de idade }\end{array}$ & $\begin{array}{l}\text { Correlações significativas entre a presente proposta } \\
\text { dos sinais prematuros e praticamente todas as prin- } \\
\text { cipais características de burnout }\end{array}$ \\
\hline $\begin{array}{l}\text { Cresswell e } \\
\text { Eklund (2005a) }\end{array}$ & $\begin{array}{l}\text { Examinar as relações entre as } \\
\text { principais características de } \\
\text { burnout, autoestima e motivação }\end{array}$ & $\begin{array}{l}\text { Jogadores profissionais de rugby } \\
(\mathrm{n}=102) \text { entre } 19 \text { e } 32 \text { anos de idade }\end{array}$ & $\begin{array}{l}\text { Jogadores com mais experiência na liga nacional } \\
\text { tendem a informar escores mais elevados de desva- } \\
\text { lorização esportiva }\end{array}$ \\
\hline $\begin{array}{l}\text { Cresswell e } \\
\text { Eklund (2005b) }\end{array}$ & $\begin{array}{l}\text { Avaliar o perfil motivacional, os } \\
\text { tipos de motivação e o perfil das } \\
\text { características do burnout }\end{array}$ & $\begin{array}{l}\text { Jogadores profissionais }(n=199), \\
\text { sexo masculino, entre } 19 \text { e } 33 \text { anos } \\
\text { de idade }\end{array}$ & $\begin{array}{l}\text { A motivação teve uma verdadeira associação com } \\
\text { as características de burnout, correlacionando-se na } \\
\text { escala com magnitude elevada para desvalorização } \\
\text { esportiva, moderada para reduzido senso de reali- } \\
\text { zação esportiva e pequena para exaustão }\end{array}$ \\
\hline $\begin{array}{l}\text { Cresswell e } \\
\text { Eklund (2005c) }\end{array}$ & $\begin{array}{l}\text { Avaliar a relação entre burnout } \\
\text { e os diferentestipos de motivação }\end{array}$ & 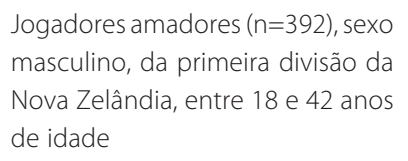 & $\begin{array}{l}\text { Os resultados sugerem níveis comparáveis de } \\
\text { burnout nas três subescalas com o estudo com atle- } \\
\text { tas colegiais dos Estados Unidos (Raedeke \& Smith, } \\
\text { 2001) }\end{array}$ \\
\hline $\begin{array}{l}\text { Cresswelle } \\
\text { Eklund (2006a) }\end{array}$ & $\begin{array}{l}\text { Observar as mudanças das prin- } \\
\text { cipais características do burnout } \\
\text { durante } 30 \text { semanas (pré-com- } \\
\text { petição, competição e final da } \\
\text { competição) }\end{array}$ & $\begin{array}{l}\text { Jogadores profissionais de rugby } \\
(n=109) \text { entre } 19 \text { e } 32 \text { anos de idade }\end{array}$ & $\begin{array}{l}\text { Aumento da frequência da experiência de senti- } \\
\text { mentos de Reduzido Senso de Realização Esportiva } \\
\text { entre a fase pré-competitiva e a competitiva } \\
\text { Nenhuma diferença estatisticamente significativa foi } \\
\text { observada em Reduzido Senso de Realização Espor- } \\
\text { tiva entre a fase pré-competitiva e o final da compe- } \\
\text { tição, e entre a fase competitiva e o fim da competição }\end{array}$ \\
\hline $\begin{array}{l}\text { Lemyre, Hall e } \\
\text { Roberts (2008) }\end{array}$ & $\begin{array}{l}\text { Investigar o burnout em atletas } \\
\text { mediante uma perspectiva } \\
\text { social-cognitiva }\end{array}$ & $\begin{array}{l}\text { Esquiadores de elite }(n=141) \text {, am- } \\
\text { bos os sexo, entre } 17 \text { e } 32 \text { anos de } \\
\text { idade }\end{array}$ & $\begin{array}{l}\text { Escores moderados para as três subescalas de } \\
\text { burnout, como para o burnout total }\end{array}$ \\
\hline Cresswell (2009) & $\begin{array}{l}\text { Identificar prematuramente os } \\
\text { sinais de burnout no meio e no } \\
\text { final da competição }\end{array}$ & $\begin{array}{l}\text { Jogadores profissionais de rugby } \\
(n=183) \text { entre } 18 \text { e } 36 \text { anos de idade }\end{array}$ & $\begin{array}{l}\text { Parte dos atletas relatou a experiência de burnout } \\
\text { nas três subescalas, em uma frequência descrita } \\
\text { como alta (Raedeke \& Smith, 2001) }\end{array}$ \\
\hline $\begin{array}{l}\text { Lonsdale et al. } \\
\text { (2009) }\end{array}$ & $\begin{array}{l}\text { Investigar as relações entre as } \\
\text { normas de comportamento e o } \\
\text { burnout }\end{array}$ & $\begin{array}{l}\text { Atletas ( } n=201 \text { ), sendo } 121 \text { do sexo } \\
\text { feminino e } 80 \text { do sexo masculino. } \\
\text { Média de idade: } 22,9 \text { anos } \\
\text { De } 51 \text { modalidades esportivas (indi- } \\
\text { viduais e coletivas) }\end{array}$ & $\begin{array}{l}\text { A autonomia e competência estão fortemente rela- } \\
\text { cionadas com os sintomas de burnout. Análises mos- } \\
\text { traram relações indiretas entre esses dois sentimentos } \\
\text { e desvalorização esportiva }\end{array}$ \\
\hline
\end{tabular}


Estudos recentes têm diferenciado burnout em atletas de treinamento excessivo ou overtraining e conceitos relacionados, que previamente foram utilizados como sinônimos. Parece haver um consenso de que burnout consiste em um estado final extremo, resultante da má adaptação ao treinamento ou recuperação incompleta (Goodger, Gorely, Lavallee \& Harwood, 2007).

\section{Considerações Finais}

Stress e burnout em atletas é um tema altamente relevante para profissionais da área esportiva. Além de investigar estressores, que podem limitar o desenvolvimento esportivo de atletas de alto nível, é importante determinar as consequências associadas ao burnout, bem como as estratégias (positivas e negativas) de enfrentamento. O enfrentamento adequado, quando empregado pelos atletas, pode minimizar o impacto do stress. Assim, a identificação de estratégias positivas permitirá delinear programas preventivos e de intervenção durante as fases de pré-competição e competição.

Outra importante questão é identificar quais os momentos de maior stress para diferentes atletas e para diferentes modalidades esportivas. Pesquisas futuras podem também investigar o papel exercido pelo tempo de experiência do atleta, que parece estar relacionado ao desenvolvimento de estratégias mais adequadas de enfrentamento. Uma possível linha de investigação é, ainda, analisar eventuais relações entre o stress psicofísico e o regime de trabalho, haja vista as alterações do sistema de trabalho ao longo das várias etapas da formação do atleta, até que ele atinja o grau profissional. Avaliar diferentes estratégias de enfrentamento, em diferentes modalidades esportivas, em curto e em longo prazo, também são questões importantes para subsidiar intervenções práticas.

\section{Referências}

Anshel, M. H., \& Si, G. (2008). Coping styles following acute stress in sport among elite Chinese athletes: a test of trait and transactional coping theories. Journal of Sport Behavior, 31 (1), 2-21.

Brasil. Ministério da Saúde. (2001). Doenças relacionadas ao trabalho: manual de procedimentos para os serviços de saúde(Série A. Normas e Manuais Técnicos). Brasília: MS.
Recuperado em novembro 23, 2009, disponível em <http://www.ims.uerj.br/espmedtrab/doenca_ trabalhador.pdf>.

Coordenação de Aperfeiçoamento de Pessoal de Nível Superior. (2009). Banco de teses. Recuperado em janeiro 10, 2010, disponível em <http://www.capes.gov.br/ servicos/banco-de-teses $>$.

Cresswell, S. L. (2009). Possible early signs of athlete burnout: a prospective study. Journal of Science and Medicine in Sport, 12 (3), 393-398.

Cresswell, S. L., \& Eklund, R. C. (2004). The athlete burnout syndrome: possible early signs. Journal of Science and Medicine in Sport, 7 (4), 481-487.

Cresswell, S. L., \& Eklund R. C. (2005a). Changes in athlete burnout and motivation over a 12- week league tournament. Medicine and Science in Sports and Exercise, 37 (11), 1957-1966.

Cresswell, S. L., \& Eklund, R. C. (2005b). Motivation and burnout among top amateur rugby players. Medicine and Science in Sports and Exercise, 37 (3), 469-477.

Cresswell, S. L., \& Eklund, R. C. (2005c). Motivation and burnout in professional rugby players. Research Quarterly for Exercise and Sport, 76 (3), 370-376.

Cresswell, S. L., \& Eklund, R. C. (2006a). The nature of player burnout in rugby: key characteristics and attributions. Journal of Applied Sport Psychology, 18 (3), 219-239.

Cresswell, S. L., \& Eklund, R. C. (2006b). The convergent and discriminant validity of burnout measures in sport: a mult-trait/multi-method analysis. Journal of Sports Sciences, 24 (2), 209-220.

Folkman, S., \& Moskowitz, J. T. (2004). Coping: pitfalls and promise. Annual Review of Psychology, 55 (1), 745-774.

Garcés de Los Fayos, E. J., \& Vives Benedicto, L. (2002). Variables motivacionales y emocionales implicadas en el síndrome de burnoud en el contexto deportivo. Revista Electrónica de Motivación y Emoción, 5 (1-2). Acceso en octubre 18, 2008, disponible en <http://reme.uji.es>.

Gimenez, M. G. G. (1997). A teoria do enfrentamento e suas implicações para sucessos e insucessos em psiconcologia. In M. G. G. Gimenez (Org.), A mulher e o câncer (pp.111-147). Campinas: Livro Pleno.

Goodger, K., Gorely, T., Lavallee, D., \& Harwood, C. (2007). Burnout in sport: a systematic review. The Sport Psychologist, 21 (2), 127-151.

Gustafsson, H., Hassmén, P., Kentta, G., \& Johansson, M. (2008). A qualitative analysis of burnout in elite Swedish athletes. Psychology of Sport and Exercise, 9 (6), 800-816.

Hanton, S., Thomas, O., \& Mellalieu, S. D. (2008). Management of competitive stress in elite sport. In B. Brewer (Ed.), International Olympic Committee Sport Psychology Handbook (pp.30-42). New York: Blackwell.

Hill, A. P., Hall, H. K., Appleton, P. R., \& Kozub, S. A. (2008). Perfectionism and burnout in junior elite soccer players: the mediating influence of unconditional self-acceptance. Psychology of Sport and Exercise, 9 (5), 630-644.

Ivarsson, A. (2008). Psychological predictors of sport injuries among soccer players. Dissertation not publication, Halmstad University, Halmstad, Sueden. 
Kavanagh, J. (2005). Stress and performance: a review of the literature and its applicability to the military. Santa Monica, CA: RAND Corporation. Retrieved May 10, 2008, from <http://www.rand.org/pubs/technical_reports/2005/ RAND_TR192.pdf>.

Lazarus, R. S. (1993). From psychological stress to the emotions: a history of changing outlooks. Annual Review of Psychology, 44 (1), 1-21.

Lazarus, R. S., \& Folkman, S. (1984). Stress, appraisal and coping. New York: Springer.

Lemyre, P. N., Hall, H. K., \& Roberts, G. C. (2008). A social cognitive approach to burnout in elite athletes. Scandinavian Journal of Medicine \& Science in Sports, 18 (2), 221-234.

Lipp, M. E. N. (2003). Mecanismos neuropsicofisiológicos do stress: teoria e aplicações. São Paulo: Casa do Psicólogo.

Lonsdale, C., Hodge, K., \& Rose, E. (2009). Athlete burnout in elite sport: a self-determination perspective. Journal of Sports Sciences, 27 (8), 785-795.

Marques, A. C. P., \& Rosado, A. F. B. (2005). Situações estressantes nos atletas de basquetebol de alta competição. Revista Brasileira de Educação Física e Esporte, 19 (1), 71-87.

Maslach, C., \& Jackson, S. E. (1981). The measurement of experienced burnout. Journal Organizational Behaviour, 2 (2), 99-113.

Maslach, C., \& Leiter, M. P. (2008). Early predictors of job burnout and engagement. Journal of Applied Psychology, 93 (3), 498-512.

Nicholls, A. R., \& Polman, R. C. J. (2007). Coping in sport: a systematic review. Journal of Sports Sciences, 25 (1), 11-31.

Nicholls, A. R., Jones, C. R., Polman, R. C. J., \& Borkoles, E. (2009). Acute sport-related stressors, coping, and emotion among professional rugby players during training and matches. Scandinavian Journal of Medicine \& Science in Sports, 19 (1), 113-120.

Noblet, A. J., \& Gifford, S. (2002). The sources of stress experienced by professional australian footballers. Journal of Applied Sport Psychology, 14 (1), 1-13.
Orlick, T. (2008). In pursuit of excellence ( $4^{\text {th }}$ ed.). Champaign, IL: Human Kinetics.

Pires, D. A., Brandão, M. R. F., \& Machado, A. A. (2005). A síndrome de burnout no esporte. Motriz, 11 (3), 147-153.

Pires, D. A., Brandão, M. R. F., \& Silva, C. B. (2006). Validação do questionário de burnout para atletas. Revista da Educação Física, 17 (1), 27-36.

Raedeke, T. D. (1997). Is athlete burnout more than just stress? A sport commitment perspective. Journal of Sport \& Exercise Psychology, 19 (4), 396-417.

Raedeke, T. D., \& Smith, A. L. (2001). Development and preliminary validation of an athlete burnout measure. Journal of Sport \& Exercise Psychology, 23 (4), 238-306.

Samulski, D. M. (2002). Psicologia do esporte. São Paulo: Manole.

Samulski, D. M., \& Lopes, M. C. (2008). Counseling Brazilian athletes during the Olympic Games in Athens 2004: important issues and intervention techniques. International Journal of Sport and Exercise Psychology, 6 (3), 277-286.

Sarafino, E. P. (2008). Health Psychology: biopsychosocial interactions ( $6^{\text {th }}$ ed.). New York: John Wiley \& Sons.

Straub, R. O. (2005). Psicologia da saúde. Porto Alegre: Artmed.

Taylor, S. E. (2009). Health psychology (7 ${ }^{\text {th }}$ ed.). Boston: McGraw-Hill.

Taylor, S. E., \& Stanton, A. L. (2007). Coping resources, coping processes, and mental health. Annual Review of Clinical Psychology, 3, 377-401.

Thatcher, J., \& Day, M. C. (2008). Re-appraising stress appraisals: the underlying properties of stress in sport. Psychology of Sport and Exercise, 9 (3), 318-335.

Weinberg, R. S., \& Gould, D. (2008). Fundamentos da psicologia do esporte e do exercício. Porto Alegre: Artmed.

Recebido em: 6/4/2010

Versão final reapresentada em: 11/1/2011

Aprovado em: 16/1/2011 
